\title{
Temporomandibular inflammation mobilizes parvalbumin and FosB/deltaFosB neurons of amygdala and dorsal raphe
}

\author{
G.C. Nascimento (i) ${ }^{1 *}$, B.B. de Paula (i) ${ }^{2 *}$, C.A. Lowry (i) ${ }^{3}$, and C.R.A. Leite-Panissi ${ }^{2}{ }^{2}$ \\ ${ }^{1}$ Departamento de Fisiologia, Faculdade de Medicina de Ribeirão Preto, Universidade de São Paulo, Ribeirão Preto, SP, Brasil \\ ${ }^{2}$ Departamento de Psicologia, Faculdade de Filosofia, Ciências e Letras de Ribeirão Preto, Universidade de São Paulo, Ribeirão \\ Preto, SP, Brasil \\ ${ }^{3}$ Department of Integrative Physiology and Center for Neuroscience, University of Colorado Boulder, Boulder, CO, USA
}

\begin{abstract}
Pathophysiological mechanisms involved in orofacial pain and their relationship with emotional disorders have emerged as an important research area for multidisciplinary studies. In particular, temporomandibular disorders (TMD) have been evaluated clinically from both physiological and psychological perspectives. We hypothesized that an altered neuronal activity occurs in the amygdala and the dorsal raphe nucleus (DR), encephalic regions involved in the modulation of painful and emotional information. Adult male Wistar rats were used in an experimental complete Freund's adjuvant (CFA)-induced temporomandibular joint (TMJ) inflammation model. CFA was applied for 1 or 10 days, and the animals were euthanized for brain samples dissection for FosB/ $\triangle$ FosB and parvalbumin (PV) immunostaining. Our results were consistent in showing that the amygdala and DR were activated in the persistent inflammatory phase (10 days) and that the expression of $\mathrm{PV}^{+}$interneurons in the amygdala was decreased. In contrast, in the DR, the expression of $\mathrm{PV}^{+}$interneurons was increased in persistent states of CFAinduced TMJ inflammation. Moreover, at 10 days of inflammation, there was an increased co-localization of $\mathrm{PV}^{+}$and FosB/ $\Delta \mathrm{FosB}^{+}$neurons in the basolateral and central nucleus of the amygdala. Different nuclei of the amygdala, as well as portions of the DR, were activated in the persistent phase (10 days) of TMJ inflammation. In conclusion, altered activity of the amygdala and DR was detected during persistent inflammatory nociception in the temporomandibular joint. These regions may be essential for both sensory and affective dimensions of orofacial pain.
\end{abstract}

Key words: Amygdala; Dorsal raphe nucleus; FosB; Parvalbumin; Temporomandibular inflammation

\section{Introduction}

One of the most common pain conditions experienced by adults is chronic orofacial pain. Specifically, among the dysfunctions that affect the stomatognathic system, temporomandibular disorder (TMD) associated with myofascial pain is a usual cause for chronic orofacial pain development (1). Preclinical and clinical studies have also elucidated the direct relationship between TMD-induced pain with emotional alterations $(2,3)$.

A consensus has formed regarding the need to clinically evaluate TMDs from both physical and psychological perspectives, and to consider both pharmacological and behavioral treatments (4). However, this idea is not yet widely applied in clinical practice due to the lack of direct preclinical and clinical experimental evidence. Indeed, the affective component of pain is mediated by circuits that are distinct from those mediating the sensorydiscriminative component (5) and research on neural mechanisms about emotional behavior in pain models can advance the knowledge on this line of research.

Cellular remodeling of brain structures is directly involved in the pathophysiology of pain-induced emotional disorders, based on findings that cortical and subcortical functional and structural neuroplastic alterations are evident in chronic pain states (6). The amygdala is intensely involved in processing the emotional component of pain, probably through a modulatory role upon major supraspinal pain control areas (7). The nociceptive inputs from extraamygdaloid areas are processed in the lateral (LA) and basolateral (BLA) nuclei of the amygdala and transmitted to the central nucleus of the amygdala (CEA), which can then

Correspondence: C.R.A. Leite-Panissi: <christie@usp.br>

${ }^{*}$ These authors contributed equally to this work. 
modulate pain through connections with the forebrain and brainstem (8). These amygdaloid nuclei are more strongly involved in processing harmful stimuli and are responsible for integrating sensory-affective information in the context of pain (9). Moreover, neurons that express the calciumbinding protein parvalbumin (PV) in the amygdala appear to be subpopulations of GABAergic local circuit neurons (10), constituting 43 and $19 \%$ of the populations of PV-positive neurons of GABA-immunoreactive neurons in the BLA and LA, respectively (11).

Also, given the critical role of serotonin in the regulation of affective functions, the serotonergic neurons of the dorsal raphe nucleus (DR) and other raphe nuclei are particularly interesting in the context of the aversion of pain (12). Projections from the DR to many areas are recognized to be involved in pain perception and pain modulation, including the spinal trigeminal nucleus (13) and amygdala (14). Importantly, the GABA interneurons are the most prevalent type of non-5-HT neurons in the $\mathrm{DR}$, occurring mainly in the ventrolateral division of the DR (15).

Regarding both sensory and affective aspects of pain, GABAergic modulation of pain transmission has been extensively investigated in the spinal cord, while its modulatory effect at the supraspinal level is scarce (16). In the context of modulatory effects of GABA signaling at supraspinal levels, the activity of PV-positive GABA ergic interneurons within the prelimbic cortex has been shown to alter sensory and emotional pain perception (17).

The inflammatory agents used in pain models range from irritant chemicals (e.g., carrageenan, formalin), microbial cell wall fragments or toxins (e.g., lipopolysaccharide), complete Freund's adjuvant (CFA), and zymosan to agents that directly activate specific receptors on primary sensory neurons (e.g., capsaicin, mustard oil). In particular, CFA has been effectively used for the persistent orofacial pain animal model associated with the temporomandibular joint $(\mathrm{TMJ})$ region $(2,18)$. The physiological factors involved and the duration of the CFA model also make it appropriate for the study of pain in psychiatric comorbidities (19). In this context, our previous results using a CFA animal model showed increased inflammatory indicators (plasma extravasation and neutrophil degranulation), as well as increased nociceptive sensitivity after $1,3,7$, and 10 days of adjuvant injection into the TMJ of rats $(2,20)$. In the same way, in that same model, an anxiety-like behavior mainly at 1 and 10 days after injections was observed (2).

In the current study, we investigated the role of CFAinduced TMJ inflammation on PV expression in the amygdala and $\mathrm{DR}$, and on neuronal activation of the same regions as assessed by FosB/ $\Delta \mathrm{FosB}$ expression. Fos-B is an immediate-early response gene, an indicator of neuronal activation, and PV is a phenotypic marker for an inhibitory interneuronal population.

\section{Material and Methods}

\section{Animals}

Adult male Wistar rats weighing 250-300 g (total number $=28$ ) that were maintained in colony conditions at $24 \pm 1^{\circ} \mathrm{C}$ on a $12-\mathrm{h}$ light/dark cycle (lights on at 06:00 $\mathrm{h}$ ) with ad libitum food and water. All experiments were approved by the Animal Care and Use Committee of the University of São Paulo, at the Ribeirão Preto campus (Brazil) (protocol \#11.1.891.53.6). The animals used did not present weight loss throughout the experiments. Moreover, the behavioral data of these animals was previously published (2).

\section{Complete Freund's adjuvant injections}

Rats were anesthetized with an intramuscular injection of $10 \%$ ketamine $(75 \mathrm{mg} / \mathrm{kg}$ ) plus $4 \%$ xylazine $(10 \mathrm{mg} / \mathrm{kg})$ followed by bilateral intraarticular administration with $50 \mu \mathrm{g}$ of CFA (Mycobacterium tuberculosis, Sigma-Aldrich, USA) suspended in $50 \mu \mathrm{L}$ paraffin oil or with $50 \mu \mathrm{L}$ of a $0.9 \%$ saline solution (SAL). CFA bilateral injections into the TMJ was adopted based on previous studies $(2,21,22)$. The following protocol for the administration of CFA into the TMJ has been described previously by do Nascimento and Leite-Panissi (2).

\section{Immunochemistry}

One or 10 days after administration of CFA bilaterally into the TMJ regions, the animals were deeply anesthetized with $10 \%$ ketamine $(225 \mathrm{mg} / \mathrm{kg}$ ) plus $4 \%$ xylazine (30 $\mathrm{mg} / \mathrm{kg}$ ) and transcardially perfused with phosphatebuffered saline (PBS) followed by $4 \%$ paraformaldehyde in $0.1 \mathrm{M}$ sodium phosphate buffer. The brains were then removed and post-fixed in 4\% paraformaldehyde for $2 \mathrm{~h}$. After that, samples were immersed in $30 \%$ sucrose in $0.1 \mathrm{M}$ sodium phosphate buffer for cryoprotection for $48 \mathrm{~h}$. Next, brains were cut serially on a cryostat (CM-1900, Leica, Germany) into $30 \mu \mathrm{m}$-thick frontal sections and again stored at $-70^{\circ} \mathrm{C}$ until immunochemistry procedure. Free-floating sections were submitted to antigen recovery (heating sections for $30 \mathrm{~min}$ in a water bath at $60^{\circ} \mathrm{C}$ in $0.1 \mathrm{M}$ citrate buffer; $\mathrm{pH}$ 6.0). After rinsing in the washing buffer (0.1 M PBS + 0.15\% Triton-X100; pH 7.4), the sections were incubated for $30 \mathrm{~min}$ at $18^{\circ} \mathrm{C}$ with $2 \%$ hydrogen peroxide (diluted in PBS + Triton) to remove endogenous peroxidase activity. Nonspecific binding sites were blocked by incubation in a solution containing $5 \%$ bovine serum albumin plus $5 \%$ normal serum (from the species of origin of the secondary antibody) in washing buffer for $1 \mathrm{~h}$ at $18^{\circ} \mathrm{C}$. Next, these sections were incubated at $18^{\circ} \mathrm{C}$ with the primary antibody for polyclonal rabbit antiFosB/ $\Delta$ FosB antibody (rabbit polyclonal, 1:1000, H-75, sc-7203, Santa Cruz Biotechnology, USA; see 23-25). This antibody recognizes both the transient FosB and the more stable $\Delta$ FosB isoforms (25). Alternatively, sections were incubated at $18^{\circ} \mathrm{C}$ with primary monoclonal mouse 
anti-PV antibody (mouse monoclonal, 1:1000, P227, Sigma-RBI), as a marker of the PV-positive subset of GABAergic interneurons. After incubation in the primary antibody for $24 \mathrm{~h}$ at $18^{\circ} \mathrm{C}$, the sections were successively washed (washing buffer) and incubated in biotinylated secondary antibody solution (goat, anti-rabbit/anti-mouse, $1: 400$, in PBS, Vector Lab, USA) for $90 \mathrm{~min}$ at $18^{\circ} \mathrm{C}$. Sections were then incubated with the avidin-biotinperoxidase complex for $2 \mathrm{~h}$ at $18^{\circ} \mathrm{C}$ (Vectastain $\mathrm{ABC}$ kit, Vector Lab), and the immunoreactivity was revealed by a peroxidase reaction using diaminobenzidine (Sigma) as the chromogen. Negative control sections were incubated at $18^{\circ} \mathrm{C}$ in the same way as described above but without primary antibodies.

\section{Double staining by immunochemistry}

A similar protocol (as described above) was used for double immunostaining with the difference of incubation of the primary antibody. The sections were primarily incubated in the first secondary antibody (rabbit anti-FosB/ $\Delta$ FosB antibody, $1: 1000$ ) for $24 \mathrm{~h}$ at $18^{\circ} \mathrm{C}$, and then washed (washing buffer) and incubated in biotinylated secondary antibody solution (1:400, in PBS) for 90 min at $18^{\circ} \mathrm{C}$. Sections were then incubated with the second primary antibody (monoclonal mouse anti-PV antibody, $1: 1000)$ for another $24 \mathrm{~h}$ at $18^{\circ} \mathrm{C}$ and again with biotinylated secondary antibody solution. Finally, sections were incubated with the avidin-biotin-peroxidase complex for $2 \mathrm{~h}$ (Vectastain $\mathrm{ABC}$ kit, Vector Lab) at $18^{\circ} \mathrm{C}$ and the immunoreactivity was revealed by a peroxidase reaction using diaminobenzidine (Sigma) as the chromogen.

\section{Quantification of immunochemistry data}

An observer blinded to the treatment conditions quantified the $\mathrm{PV}^{+}$or FosB/ $\mathrm{F}$ FosB ${ }^{+}$cells immunolabeling. Digital images were obtained in a Leica microscope. Each image was acquired with its bar scale. For quantification, five images were systematically captured for each brain region studied with a $20 \times$ objective. Immunoreactive cells were counted using ImageJ software (Research Services Branch, National Institutes of Health, USA). First, a background measure was performed, and this value was removed from the final quantification of all photomicrographs.

Cells with gray (FosB $\left./ \Delta \mathrm{FosB}^{+}\right)$or brown $\left(\mathrm{PV}^{+}\right)$impregnations with areas between 10 and $80 \mu \mathrm{m}^{2}$ were considered for the counting. Neuroanatomical sites were identified with the help of a stereotaxic atlas of the rat brain by Paxinos and Watson (26).

For double staining quantification, five images from the complete serial section were selected randomly. The number of cells simultaneously stained with FosB/AFosB ${ }^{+}$ and $\mathrm{PV}^{+}$in the same areas were counted under microscopy visualization. FosB/ $\Delta$ FosB and PV double-labeled neurons were counted, and the proportion of double-labeling neurons to total FosB/ $\Delta$ FosB neurons (as percentage) was then calculated.
The anterior-posterior localization from bregma of the analyzed regions were: BLA, CEA, and LA nuclei of the amygdala, -1.72 to $-3.24 \mathrm{~mm}$ bregma; dorsal division of the dorsal raphe nucleus (DRD), -6.84 to $-6.96 \mathrm{~mm}$ bregma; ventral division of the dorsal raphe nucleus (DRV), -7.20 to $-8.40 \mathrm{~mm}$ bregma; and lateral division of the dorsal raphe nucleus (DRL), -7.20 to $-8.04 \mathrm{~mm}$ bregma.

\section{Statistical analysis}

Statistical differences between means were calculated using a two-way ANOVA for repeated measures followed by the post hoc Newman-Keuls test, with treatment and time as factors for statistical analysis. Data were considered statistically significant when $\mathrm{P}<0.05$.

\section{Results}

The effects of CFA-induced TMJ inflammation on FosB/AFosB expression in the distinct nuclei of the amygdala and DR are presented in Figure 1. In the BLA, CFA injections led to a significant increase of FosB/ $\Delta$ FosB positive neurons. The group with 10 days of CFA-induced inflammation was different compared with Day 10/saline and Day 1/CFA-induced inflammation groups $(P<0.05$, Figure 1B). The LA analysis also revealed an increase in FosB $/ \Delta$ FosB $^{+}$neurons in the group with 10 days of CFAinduced inflammation and was different compared with Day 10/saline and Day 1 of CFA-induced inflammation groups $(P<0.05$, Figure $1 C)$. However, no difference was found in $\mathrm{FosB} / \Delta \mathrm{FosB}^{+}$neurons in the CEA among groups (Figure 1D).

In the DRL, we found significant effects on the number of $\mathrm{FosB} / \Delta \mathrm{FosB}^{+}$neurons in the group with 10 days of CFA-induced inflammation compared with Day 10/saline and Day 1/CFA-induced inflammation groups $(P<0.05$, Figure 1F). Similarly, in the DRD, an increase of FosB/ $\triangle \mathrm{FosB}^{+}$neurons in the group with 10 days of CFAinduced inflammation was found compared with Day 10/ saline and Day 1 of CFA-induced inflammation groups $(P<0.05$, Figure $1 G)$. FosB $/ \Delta F_{0 s}{ }^{+}$neurons in the $D R V$ showed a significant increase in the group with 10 days of CFA-induced inflammation compared with Day 10/saline and Day 1 of CFA-induced inflammation groups $(P<0.05$, Figure $1 \mathrm{H}$ ).

The amygdala and DR analyses revealed that CFAinduced TMJ inflammation produced an alteration of $\mathrm{PV}^{+}$ interneuron expression (Figure 2). Among $\mathrm{PV}^{+}$neuron counts in the BLA, we found a significant reduction of the group with 10 days of CFA-induced inflammation compared with Day 10/saline and Day 1 of CFA-induced inflammation groups $(P<0.05$, Figure $2 B$ ). Interestingly, in the LA analysis, the group with 1 day of CFA-induced inflammation showed more $\mathrm{PV}^{+}$neurons compared with the Day 1/saline group. However, 10 days of CFA-induced inflammation lead to a decrease in the $\mathrm{PV}^{+}$neurons compared with Day 1 of CFA-induced inflammation group 

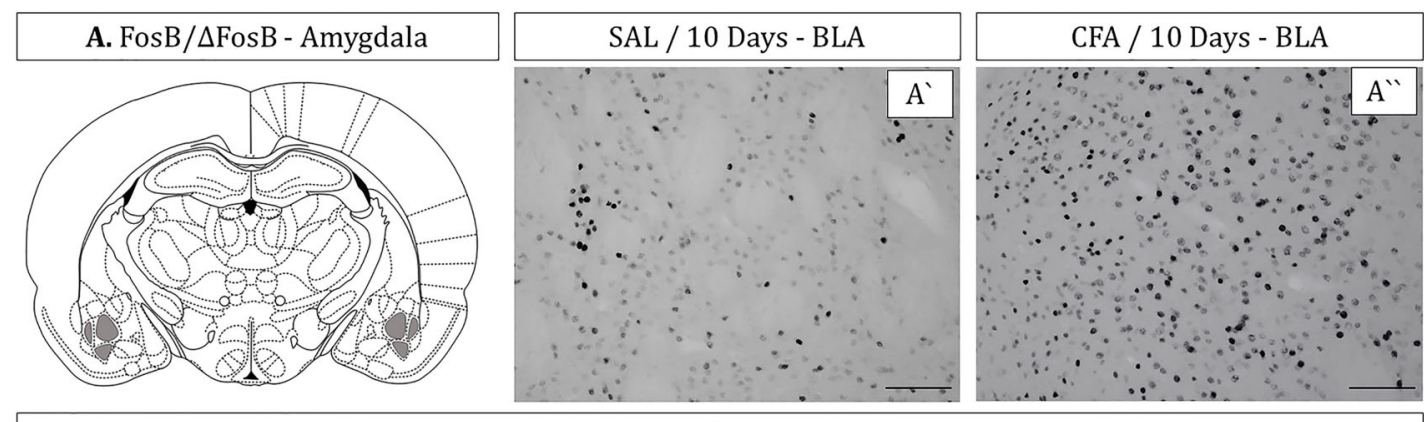

$\operatorname{BREGMA}(\mathrm{mm}):-2.40$
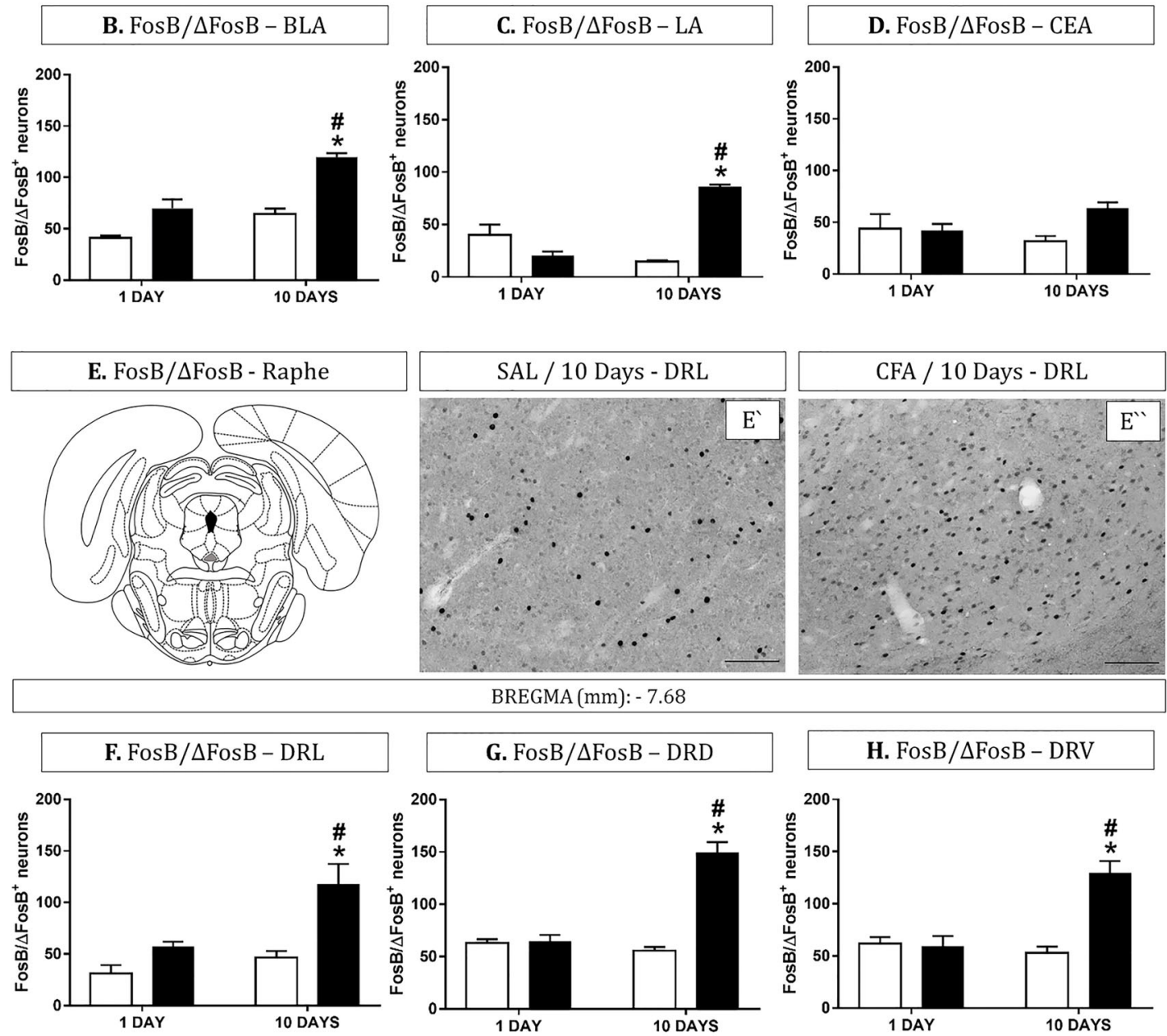

BREGMA (mm): - 7.68

G. FosB $/ \Delta$ FosB - DRD

H. FosB $/ \triangle$ FosB $-\mathrm{DRV}$
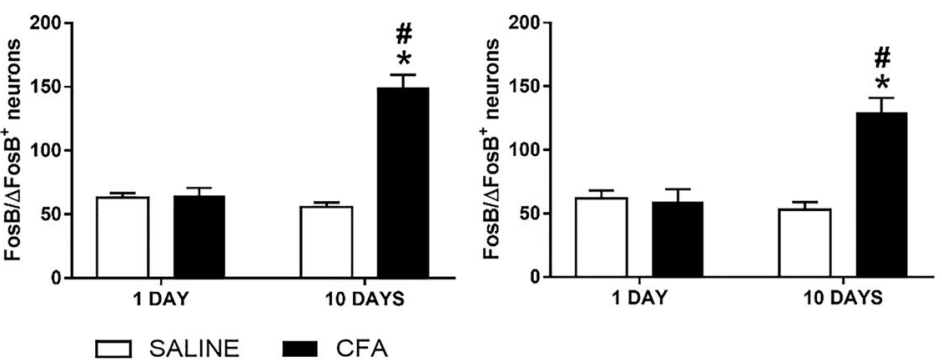

Figure 1. Subregions of the amygdala and dorsal raphe nucleus were analyzed for neuronal activation in complete Freund's adjuvant (CFA)-induced temporomandibular inflammation ( 1 or 10 days) and rats administered saline (SAL) ( $n=6-7$ per group). A, Plate adapted from Paxinos and Watson Atlas (26; Elsevier Academic Press) of basolateral nucleus of the amygdala (BLA); $\mathbf{A}^{\prime}$, photomicrograph of FosB/ $\triangle$ FosB staining in the BLA of a representative rat from the Day 10/saline-treated group and A", from the Day 10/CFA-treated group. Graphs show FosB/AFosB immunohistochemistry (IR) quantification in BLA - B, lateral (LA - C), and central nuclei of the amygdala (CEA - D) (-2.40 mm bregma). E, Plate adapted from Paxinos and Watson Atlas (26; Elsevier Academic Press) of the ventral division of the dorsal raphe (DRV); E', photomicrograph of FosB/AFosB IR in the DRL of a representative rat from the Day 10/saline treated group and E', from the Day 10/CFA treated group. Graphs show FosB/ $\triangle$ FosB IR quantification in lateral (DRL - F), dorsomedial (DRD - G), and ventral divisions (DRV - H) of the dorsal raphe nucleus $\left(-7.68\right.$ bregma). Data are reported as means $\pm S E$. ${ }^{*} P<0.05 v s$ Day10/control group (saline), ${ }^{\#} \mathrm{P}<0.05$ vs Day 1/CFA group (Newman-Keuls test). Magnification, $20 \times$; scale bars, $100 \mu \mathrm{m}$. 

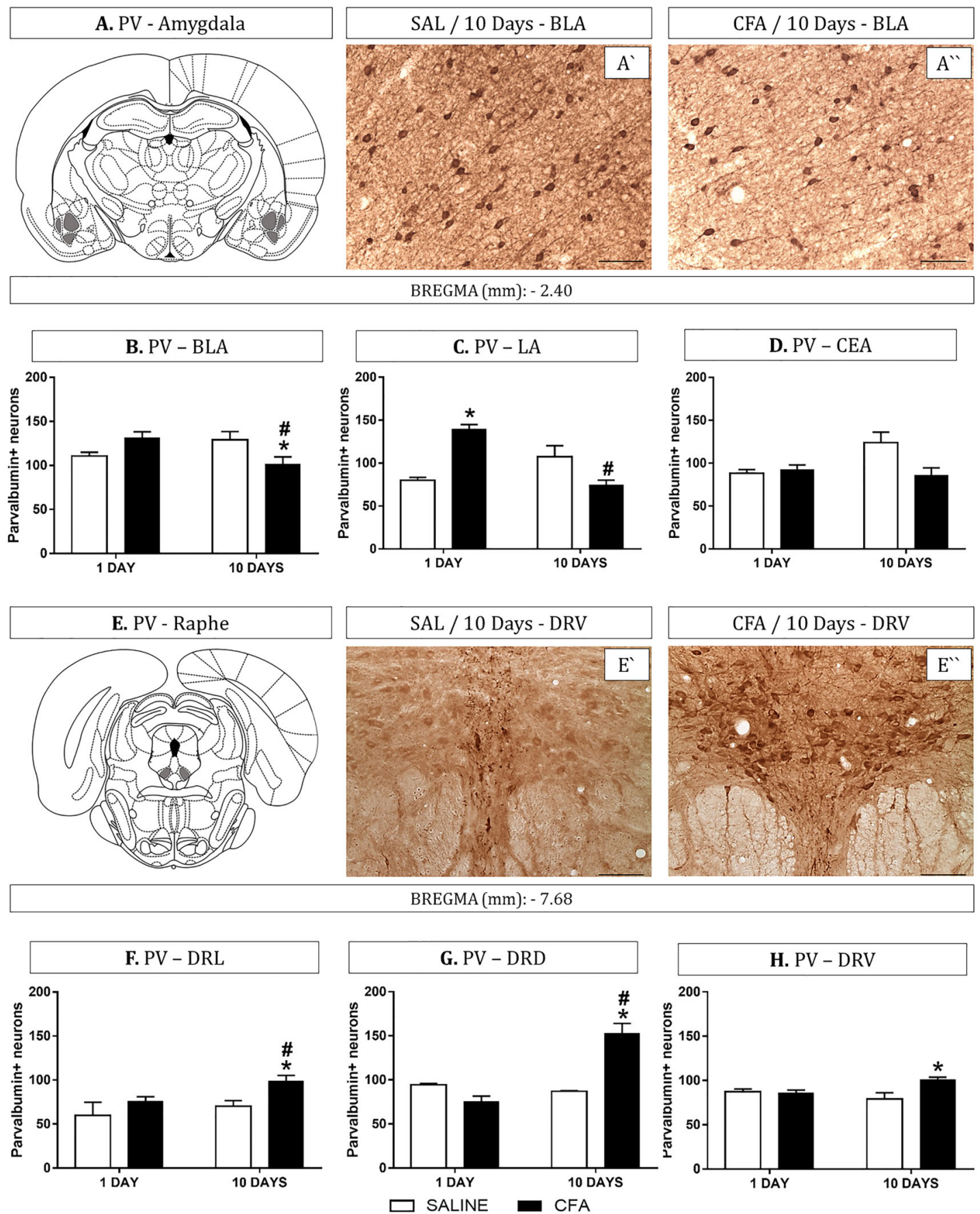

Figure 2. Subregions of the amygdala and dorsal raphe nucleus were analyzed for parvalbumin (PV) expression in complete Freund's adjuvant (CFA)-induced temporomandibular inflammation (1 or 10 days) and rats administered saline (SAL) ( $n=6-7$ per group). A, Plate adapted from Paxinos and Watson Atlas (26; Elsevier Academic Press) of basolateral nuclei of the amygdala (BLA); A', photomicrograph of PV staining in the BLA of a representative rat from the Day 10/saline-treated group and A', from the Day 10/ CFA treated group. Graphs show PV immunohistochemistry (IR) quantification in basolateral (BLA - B), lateral (LA - C), and central nuclei of the amygdala (CEA - D) (-2.40 mm bregma). E, Plate adapted from Paxinos and Watson Atlas (26; Elsevier Academic Press) of the lateral division of the dorsal raphe nucleus (DRL); E', photomicrograph of PV IR in the DRV of a representative rat from the Day 10/saline-treated group and E', from the Day 10/CFA-treated group. Graphs show PV IR quantification in lateral (DRL - F), dorsal (DRD - G), and ventral (DV - H) divisions of the dorsal raphe nucleus $\left(-7.68 \mathrm{~mm}\right.$ bregma). Data are reported as means $\pm \mathrm{SE}$. ${ }^{*} \mathrm{P}<0.05 \mathrm{vs} \mathrm{Day}$ $10 /$ control group (saline), ${ }^{\#} \mathrm{P}<0.05$ vs Day 1/CFA group (Newman-Keuls test). Magnification, $20 \times$; scale bars, $100 \mu \mathrm{m}$. 
$(P<0.05$, Figure 2C). In the CEA, there was no significant effect on $\mathrm{PV}^{+}$neurons among the groups $(\mathrm{P}<0.05$, Figure 2D).

In the DRL, CFA significantly increased the number of $\mathrm{PV}^{+}$neurons in the group with 10 days of CFA-induced inflammation and was different compared with the Day 10/ saline group $(P<0.05$, Figure $2 F)$. In the DRD, 10 days of CFA-induced inflammation significantly increased the $\mathrm{PV}^{+}$neurons compared with Day 10/saline and Day 1 of CFA-induced inflammation groups $(P<0.05$, Figure $2 \mathrm{G})$. Regarding DRV, CFA increased the $\mathrm{PV}^{+}$neuron counts in the group with 10 days of CFA-induced inflammation compared with the Day 10/saline group $(P<0.05$, Figure $2 \mathrm{H}$ ).

Double staining of FosB $/ \Delta$ FosB and $P V$ neurons were made in the amygdala and DR (Figure 3 ). In the BLA, the group with 10 days of CFA-induced inflammation showed an increase in the FosB/AFosB and $\mathrm{PV}^{+}$neurons compared with Day 10/saline and Day 1/CFA-induced inflammation groups $(P<0.05$, Figure $3 C)$. Moreover, the CEA analysis also revealed an increase in the FosB $/ \triangle \mathrm{FosB}^{+}$ and $\mathrm{PV}^{+}$neurons in the group with 10 days of CFAinduced inflammation compared with Day 10/saline and Day 1 of CFA-induced inflammation groups $(P<0.05$, Figure 3E). Additionally, no effect was found in the co-localization of $\mathrm{PV}^{+}$and FosB/ $\Delta \mathrm{FosB}^{+}$neurons on $\mathrm{LA}$ and DR (Figure 3D and $\mathrm{H}-\mathrm{J}$ ).

\section{Discussion}

Our findings showed that: i) different nuclei of the amygdala, as well as portions of the DR, were activated in the persistent phase (10 days) of TMJ inflammation; ii) the expression of $\mathrm{PV}^{+}$interneurons in the amygdala was decreased while in the DR it was increased in persistent states of CFA-induced TMJ inflammation; and iii) in the acute phase of CFA-induced TMJ inflammation (i.e., Day $1)$, these changes were not detected.

Indeed, chronic pain is a complex web of sensory and emotional experiences, coupled with behavioral adaptations (27) and, in particular, chronic orofacial pain involves risk factors such as stress and depression, as well as physical dysfunction (28). Psychological stress that perpetuates and worsens is a risk factor for temporomandibular disorders of muscular or articular origin (29).

We demonstrated here that the two structures frequently recruited in the emotional and affective dimensions of nociception suffer alterations in the persistent phase of CFA-induced TMJ inflammation, an established experimental model for temporomandibular inflammatory nociception as reported previously (2). Importantly, changes in the amygdala neural circuit have been detected in different preclinical models of pain and linked mechanistically to pain behaviors, suggesting that these maladaptive neuroplastic changes are a brain mechanism of pain (7).
CFA-induced chronic pain is a potent experimental model for investigations about specific neural circuits remodeling involved in chronic pain and its comorbidity (30). Recent findings (30) suggest that the nociceptive stimuli evoked by CFA intraplantar inflammation for 7 days could weaken the excitatory glutamatergic information to the indirect pathway within the reward circuits (nucleus accumbens), indicating that the mesolimbic reward circuitry is critical for pain sensation and pain-related emotional experiences. Again, the CFA-injected mouse model is used to induce pain and anxiety-like behaviors synchronously. In accordance, Guan et al. (31) administered CFA in the hind paw to produce pain- and anxiety-like behaviors, and 7 days later, they observed the anxiolytic effects of polydatin by suppressing inflammatory cytokines in the amygdala in this model. Corroborating such findings, our previous data are consistent in suggesting the CFA-experimental model for temporomandibular inflammatory nociception for 10 days as generating an anxiolytic-like response (2).

Highly processed information generated in the LA-BLA network is transmitted to the CEA, which serves major amygdala output functions and is a locus of the descending inhibitory pathway, through forebrain and brainstem connections (32). Of note, it has been shown that the activated neurons in the CEA allow the descending inhibitory pain pathway to be disinhibited (33). Accordingly, our data showed an increased FosB/ $\Delta$ FosB expression in the CEA at 10 days of TMJ inflammation. Importantly, excitatory neurons in the cortex are under strong regulation by GABAergic interneurons, and previous findings have shown that optogenetic stimulation of one class of GABAergic interneurons, i.e., PV-containing interneurons, is sufficient to have a substantial effect on pain thresholds (34). In the study by Kang and colleagues (34), the stimulation of this single interneuron class mimicked the inhibition effects of the excitatory neurons within this brain structure during painful stimuli, and PV activation was able to alleviate mechanical hypersensitivity. Our results corroborate these findings implicating the $\mathrm{PV}^{+}$subset of GABAergic interneurons in control of chronic pain since we found a decreased expression of $\mathrm{PV}^{+}$neurons after 10 days of TMJ inflammation compared to control and 1 day of inflammation in the BLA, and after 1 day of inflammation in the LA. However, we did not find a reduction of the $\mathrm{PV}^{+}$neurons in the CEA after 10 days of TMJ inflammation; the percentage of co-localized $\mathrm{PV}^{+}$and FosB/ $\Delta$ FosB $^{+}$neurons were higher in the 10 days of TMJ inflammation compared to controls and after 1 day of inflammation in CEA and BLA. It has been shown that one week following peripheral nerve injury was enough to decrease PV interneuron activity in the somatosensory cortex (35), and the optogenetic activation of these cells promoted a reduction of the CFA-induced mechanical hypersensitivity (34). Our results are consistent with these studies since we found that 10 days of CFA-induced inflammation led to a 

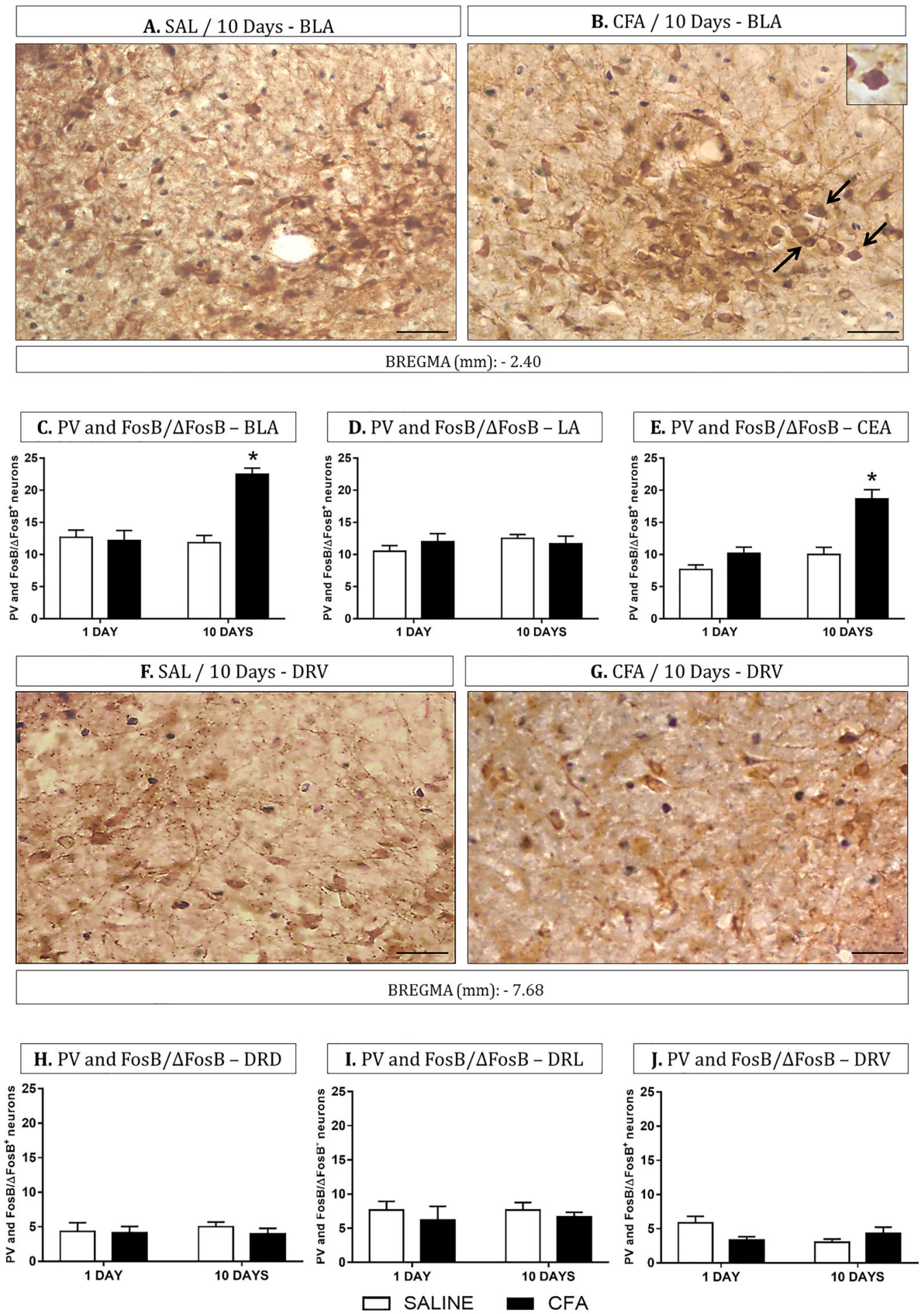

Figure 3. Representative photomicrographs of co-localization of FosB/ $\Delta$ FosB over the entire neuronal population of parvalbumin (PV), or unlabeled cells in the amygdala $(\mathbf{A}-\mathbf{E})$ and dorsal raphe $(\mathbf{F}-\mathbf{J})(\mathrm{n}=6$ per group). SAL: saline; CFA: complete Freund's adjuvant. (C-E) Percent of co-localized PV and FosB/AFosB ${ }^{+}$neurons in basolateral (BLA), lateral (LA), and central nuclei of the amygdala (CEA). (H-J) Percent of co-localized PV and FosB $/ \triangle \mathrm{FosB}^{+}$neurons in the dorsal (DRD), lateral (DRL), and ventral (DRV) divisions of the dorsal raphe nucleus. Black arrows indicate FosB $/ \Delta \mathrm{FosB}^{+}$(gray nucleus), $\mathrm{PV}^{+}$(brown cell body), and unlabeled (cell body not labeled) neurons. Data are reported as means \pm SE. ${ }^{*} P<0.05$ vs Day $1 / C F A$ group and Day 10/control group (saline) (Newman-Keuls test). Magnification, $40 \times ;$ scale bars, $100 \mu$ m. 
reduction of $\mathrm{PV}^{+}$neurons in the BLA and LA. However, the increase of the co-localization of the $\mathrm{PV}^{+}$and FosB/ $\triangle \mathrm{FosB}^{+}$neurons in the BLA and CEA shows that these interneurons are being recruited in the chronic phase of TMJ inflammation.

The brainstem DR is involved in the descending pathway that controls noxious inputs to the spinal cord, as well as noxious inputs to the trigeminal complex (36), and it participates in the normal physiological responses to noxious stimulation (37). This inhibition of the nociceptive responses might be partly achieved by direct modulation of spinal/trigeminal nociceptive transmission by the DR (38). The activation of this structure during orofacial inflammation, as shown by our results, demonstrated the adaptation of this complex system in response to chronic orofacial inflammation and may suggest an ineffective nociception modulation. In respect to the role of the DR in pain modulation, functionally, GABAergic signaling mechanisms have been described to be hyperalgesic in this region, since microinjection of a GABA receptor agonist into the DR antagonizes the analgesia produced by morphine microinjected at the same site (38). Our data were consistent in showing increased expression of $\mathrm{PV}^{+}$ neurons in the DR in the persistent phase of CFA-induced TMJ inflammation, indicating that these inhibitory interneurons in these structures may be involved in orofacial inflammation modulation.

An important point to be considered is the neuroplasticity of brain function and structure that individuals who

\section{References}

1. Calderon PS, Hilgenberg PB, Rossetti LM, Laurenti JV, Conti PC. Influence of tinnitus on pain severity and quality of life in patients with temporomandibular disorders. J Appl Oral Sci 2012; 20: 170-173, doi: 10.1590/S1678-7757 2012000200008.

2. do Nascimento GC, Leite-Panissi CRA. Time-dependent analysis of nociception and anxiety-like behavior in rats submitted to persistent inflammation of the temporomandibular joint. Physiol Behav 2014; 125: 1-7, doi: 10.1016/j. physbeh.2013.11.009.

3. Monaco A, Cattaneo R, Marci MC, Pietropaoli D, Ortu E. Central sensitization-based classification for temporomandibular disorders: a pathogenetic hypothesis. Pain Res Manag 2017; 2017: 5957076, doi: 10.1155/2017/5957076.

4. Fillingim RB, Slade GD, Greenspan JD, Dubner R, Maixner $W$, Bair $E$, et al. Long-term changes in biopsychosocial characteristics related to temporomandibular disorder: findings from the OPPERA study. Pain 2018; 159: 2403-2413, doi: 10.1097/j.pain.0000000000001348.

5. Auvray M, Myin E, Spence C. The sensory-discriminative and affective-motivational aspects of pain. Neurosci Biobehav Rev 2010; 34: 214-223, doi: 10.1016/j.neubiorev. 2008.07.008.

6. Simons LE, Elman I, Borsook D. Psychological processing in chronic pain: a neural systems approach. Neurosci experience chronic orofacial pain can present. Brain neuroimaging has helped the investigation on this topic $(39,40)$. The prefrontal cortex, the limbic system, and the circuitry of reward processing appear to be functionally and structurally altered during chronic orofacial pain (40). The neural mechanisms of such adaptive alterations in brain organization regarding temporomandibular chronic inflammation are still unclear. Our data add to the information on molecular changes in the amygdala and raphe that may be indicative of such plasticity and can contribute to brain markers of chronic orofacial pain.

For the first time, we showed the neuronal activation of the different nuclei of the amygdala and DR on TMJ inflammation. This finding can help clarify the different neural networks and will be essential for the future development of novel therapies for treating physical and emotional aspects of orofacial pain disorders in patients.

\section{Acknowledgments}

The authors thank Jared D. Heinze for technical support. This work was supported by Conselho Nacional de Pesquisa, Brazil (CNPq \#304215/2016-3), Coordenação de Aperfeiçoamento de Pessoal de Nível Superior, Brazil (Capes PDSE Program, Finance Code 001), and Fundação de Amparo à Pesquisa do Estado de São Paulo, Brazil (FAPESP \#11/07718-9). C.R.A. LeitePanissi received a research fellowship from $\mathrm{CNPq}$ (\#309215/2019-6).
Biobehav Rev 2014; 39: 61-78, doi: 10.1016/j.neubiorev. 2013.12.006.

7. Veinante $\mathrm{P}$, Yalcin I, Barrot M. The amygdala between sensation and affect: a role in pain. $J$ Mol Psychiatry 2013; 1: 9, doi: 10.1186/2049-9256-1-9.

8. Neugebauer V Li W, Bird GC, Han JS. The amygdala and persistent pain. Neuroscientist 2004; 10:221-234, doi: $10.1177 / 1073858403261077$.

9. Neugebauer V. Amygdala pain mechanisms. Handb Exp Pharmacol 2015; 227: 261-284, doi: 10.1007/978-3-66246450-2.

10. Yilmazer-Hanke DM, Faber-Zuschratter $H$, Linke $R$, Schwegler $\mathrm{H}$. Contribution of amygdala neurons containing peptides and calcium-binding proteins to fear-potentiated startle and exploration-related anxiety in inbred Roman highand low-avoidance rats. Eur J Neurosci 2002; 15: 12061218, doi: 10.1046/j.1460-9568.2002.01945.x.

11. McDonald A, Mascagni F. Colocalization of calcium-binding proteins and GABA in neurons of the rat basolateral amygdala. Neuroscience 2001; 105: 681-693, doi: 10.1016/ S0306-4522(01)00214-7.

12. Nakamura K, Shirane M, Koshikawa N. Site-specific activation of dopamine and serotonin transmission by aniracetam in the mesocorticolimbic pathway of rats. Brain Res 2001; 897: 82-92, doi: 10.1016/S0006-8993(01)02096-0. 
13. Matsuzaki S, Takada M, Li YQ, Tokuno $H$, Mizuno N. Serotoninergic projections from the dorsal raphe nucleus to the nucleus submedius in the rat and cat. Neuroscience 1993; 55:403-416, doi: 10.1016/0306-4522(93)90509-E.

14. Vianna DM, Brandão ML. Anatomical connections of the periaqueductal gray: specific neural substrates for different kinds of fear. Braz J Med Biol Res 2003; 36: 557-566, doi: 10.1590/S0100-879X2003000500002.

15. Calizo LH, Akanwa A, Ma XH, Pan YZ, Lemos JC, Craige C, et al. Raphe serotonin neurons are not homogenous: electrophysiological, morphological and neurochemical evidence. Neuropharmacology 2011; 61: 524-543, doi: 10.1016/j.neuropharm.2011.04.008.

16. Narita M, Niikura K, Nanjo-Niikura K, Narita M, Furuya M, Yamashita A, et al. Sleep disturbances in a neuropathic pain-like condition in the mouse are associated with altered GABAergic transmission in the cingulate cortex. Pain 2011; 152: 1358-1372, doi: 10.1016/j.pain.2011.02.016.

17. Zhang Z, Gadotti VM, Chen L, Souza IA, Stemkowski PL, Zamponi GW. Role of prelimbic GABAergic circuits in sensory and emotional aspects of neuropathic pain. Cell Rep 2015; 12: 752-759, doi: 10.1016/j.celrep.2015.07.001.

18. Desiderá AC, Nascimento GC, Gerlach RF, Leite-Panissi CRA. Laser therapy reduces gelatinolytic activity in the rat trigeminal ganglion during temporomandibular joint inflammation. Oral Dis 2015; 21: 652-658, doi: 10.1111/odi.12330.

19. Parent AJ, Beaudet $N$, Beaudry $H$, Bergeron J, Bérubé $P$, Drolet $\mathrm{G}$, et al. Increased anxiety-like behaviors in rats experiencing chronic inflammatory pain. Behav Brain Res 2012; 229: 160-167, doi: 10.1016/j.bbr.2012.01.001.

20. Nascimento GC, Rizzi E, Gerlach RF, Leite-Panissi. Expression of MMP-2 and MMP-9 in the rat trigeminal ganglion during the development of temporomandibular joint inflammation. Braz J Med Biol Res 2013; 46: 956-967, doi: 10.1590/1414-431X20133138.

21. Spears R, Dees LA, Sapozhnikov M, Bellinger LL, Hutchins $B$. Temporal changes in inflammatory mediator concentrations in an adjuvant model of temporomandibular joint inflammation. J Orofac Pain 2005; 19: 34-40.

22. Harper RP, Kerins CA, Mclntosh JE, Spears R, Bellinger LL. Modulation of the inflammatory response in the rat TMJ with increasing doses of complete Freund's adjuvant. Osteoarthritis Cartilage 2001; 7: 619-624, doi: 10.1053/joca.2001.0461.

23. Hiroi N, Brown JR, Haile CN, Ye H, Greenberg ME, Nestler EJ. FosB mutant mice: loss of chronic cocaine induction of Fos-related proteins and heightened sensitivity to cocaine's psychomotor and rewarding effects. Proc Natl Acad Sci USA 1997; 94: 10397-10402, doi: 10.1073/pnas.94.19.10397.

24. Hiroi N, Martín AB, Grande C, Alberti I, Rivera A, Moratalla R. Molecular dissection of dopamine receptor signaling. J Chem Neuroanat 2002; 23: 237-242, doi: 10.1016/S08910618(02)00010-8.

25. Grande $\mathrm{C}$, Zhu H, Martin AB, Lee M, Ortiz O, Hiroi N, et al. Chronic treatment with atypical neuroleptics induces striosomal FosB/DFosB expression in rats. Biol Psychiatry 2004; 55: 457-463, doi: 10.1016/j.biopsych.2003.08.008.

26. Paxinos $\mathrm{G}$, Watson $\mathrm{C}$. The rat brain in stereotaxic coordinate, the new coronal set. 5th ed. New York, Elsevier Academic Press; 2005.

27. Vachon-Presseau E, Centeno MV, Ren W, Berger SE, Tétreault $P$, Ghantous $M$, et al. The emotional brain as a predictor and amplifier of chronic pain. J Dent Res 2016; 95: 605-612, doi: 10.1177/0022034516638027.

28. Slade GD, Ohrbach R, Greenspan JD, Fillingim RB, Bair E, Sanders $\mathrm{AE}$, et al. Painful temporomandibular disorder: a decade of discovery from OPPERA studies. J Dent Res 2016; 95: 1084-1092, doi: 10.1177/0022034516653743.

29. Fernández RAR, Pereira YCL, lyomasa DM, Calzzani RA, Leite-Panissi CRA, lyomasa MM, et al. Metabolic and vascular pattern in medial pterygoid muscle is altered by chronic stress in an animal model of hypodontia. Physiol Behav 2018; 185: 70-78, doi: 10.1016/j.physbeh.2017.12.026.

30. Qi C, Guo B, Ren K, Yao H, Wang M, Sun T, et al. Chronic inflammatory pain decreases the glutamate vesicles in presynaptic terminals of the nucleus accumbens. Mol Pain 2018; 14: 1744806918781259, doi: 10.1177/174480691878 1259.

31. Guan SY, Zhang K, Wang XS, Yang L, Feng B, Tian DD, et al. Anxiolytic effects of polydatin through the blockade of neuroinflammation in a chronic pain mouse model. Mol Pain 2020; 16: 1744806919900717, doi: 10.1177/17448069199 00717.

32. Butler RK, Oliver EM, Fadel JR, Wilson MA. Hemispheric differences in the number of parvalbumin-positive neurons in subdivisions of the rat basolateral amygdala complex. Brain Res 2018; 1678: 214-219, doi: 10.1016/j.brainres.2017. 10.028 .

33. Butler RK, Ehling S, Barbar M, Thomas J, Hughes MA, et al. Distinct neuronal populations in the basolateral and central amygdala are activated with acute pain, conditioned fear, and fear-conditioned analgesia. Neurosci Lett 2017; 661: 11-17, doi: 10.1016/j.neulet.2017.09.025.

34. Kang SJ, Kwak C, Lee J, Sim SE, Shim J, Choi T, et al. Bidirectional modulation of hyperalgesia via the specific control of excitatory and inhibitory neuronal activity in the ACC. Mol Brain 2015; 8: 81, doi: 10.1186/s13041-015-0170-6.

35. Cichon J, Blanck TJJ, Gan WB, Yang G. Activation of cortical somatostatin interneurons prevents the development of neuropathic pain. Nat Neurosci 2017; 20: 11221132, doi: 10.1038/nn.4595.

36. de Leeuw R, Albuquerque R, Okeson J, Carlson C. The contribution of neuroimaging techniques to the understanding of supraspinal pain circuits: implications for orofacial pain. Oral Surg Oral Med Oral Pathol Oral Radiol Endod 2005; 100: 308-314, doi: 10.1016/j.tripleo.2004.11.014.

37. Prado WA, Faganello FA. The anterior pretectal nucleus participates as a relay station in the glutamate-, but not morphine-induced antinociception from the dorsal raphe nucleus in rats. Pain 2000; 88: 169-176, doi: 10.1016/ S0304-3959(00)00326-2.

38. Wang QP, Nakai Y. The dorsal raphe: an important nucleus in pain modulation. Brain Res Bull 1994; 34: 575-585, doi: 10.1016/0361-9230(94)90143-0.

39. Gerstner GE, Gracely RH, Deebajah A, Ichesco E, Quintero A, Clauw DJ, et al. Posterior insular molecular changes in myofascial pain. J Dent Res 2012; 91: 485-490, doi: 10.1177/0022034512443366.

40. Lin CS. Brain signature of chronic orofacial pain: a systematic review and meta-analysis on neuroimaging research of trigeminal neuropathic pain and temporomandibular joint disorders. PLoS One 2014, 23; 9: e94300, doi: 10.1371/journal.pone.0094300. 